\title{
Phosphorus doped and defects engineered graphene for improved electrochemical sensing: synergistic effect of dopants
}

\section{and defects}

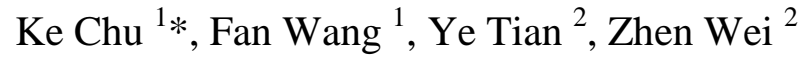

${ }^{1}$ School of Mechatronic Engineering, Lanzhou Jiaotong University, Lanzhou 730070, China

${ }^{2}$ College of Science, Hebei North University, Zhangjiakou 075000, China

*Corresponding author. E-mail address: chukelut@163.com (K. Chu) 


\begin{abstract}
Heteroatom-doped graphene materials emerged as promising metal-free catalysts have recently attracted a growing interest in electrochemical sensing applications. However, their catalytic activity and sensing performances still need to be further improved. Herein, we reported the development of unique phosphorus (P)-doped and plasma-etched graphene (denoted as PG-E) as an efficient metal-free electrocatalyst for dopamine (DA) sensing. It was demonstrated that introducing both P-dopants and plasma-engineered defects in graphene could synergistically improve the activity toward electrocatalytic oxidation of DA by increasing the accessible active sites and promoting the electron transport capability. The resulting PG-E modified electrode showed exceptional DA sensing performances with low detection limit, high selectivity and good stability. These results suggested that the synergistic effect of dopants and defects might be an important factor for developing the advanced graphene-based metal-free catalysts for electrochemical sensing.
\end{abstract}

Keywords: Heteroatom-doped graphene; Phosphorus doping; Defect-engineering; Dopamine detection; Electrochemical sensor. 


\section{Introduction}

Owing to the pronounced advantages of simplicity, fast response, good sensitivity and low cost, electrochemical sensor as an effective analytical tool has been widely used in environmental protection, food safety, clinical diagnostics and biological/pathological analysis $[1,2]$. Since the performance of the electrochemical sensors relies strongly on the activity of the electrode catalysts[3], the development of cost-effective and efficient catalysts is of paramount importance. Albeit the precious metals such as $\mathrm{Pt}, \mathrm{Pd}, \mathrm{Au}, \mathrm{Ag}$ and their alloys are the most active catalysts for electrochemical sensing [4], they have the major drawbacks of limited reserves, high cost and insufficient durability. To address these issues, tremendous progress has been made to develop transition metal-based catalysts such as $\mathrm{Co}, \mathrm{Mn}, \mathrm{Ni}, \mathrm{Cu}$ and their oxides as alternatives to precious metals due to their low cost and comparable eletrocatalytic activity [5]. However, transition metal-based catalysts suffer from low electrical conductivity and problematic dissolution susceptibility to acidic/basic electrolytes. Therefore, these disadvantages of metal-based catalysts impeded their large-scale commercial applications, and the metal-free catalysts hold great promise for electrochemical sensing but are still very challenging.

Heteroatom-doped carbonaceous materials, including carbon nanotubes, carbon nanofibers, porous carbons and graphene have gained noticeable popularity as metal-free electrocatalysts for electrochemical fields [6-8]. Among them, graphene has received the most attention because of its unique 2D structures, earth-abundant raw materials and ease of functionalizations[9]. Graphene naturally shows a high 
degree of chemical inertness due to its zero band gap, while introduction of heteroatoms $(\mathrm{N}, \mathrm{B}, \mathrm{S}, \mathrm{P}$, etc.) into graphene frameworks can tailor the electronic structures to show dramatically enhanced catalytic activity because the introduced heteroatoms enable the activation of their adjacent carbon atoms to considerably alter the charge population and spin density [8]. Furthermore, co-doping of two kinds of heteroatoms (e.g., $\mathrm{N}$ and $\mathrm{S}$ ) can further increase the catalytic activity as compared to their single-doped counterparts attributed to a synergistic coupling effect of two heteroatoms [7]. Currently, the graphene-based metal-free materials have been intensively studied in energy-relevant electrochemical applications, such as fuel cells [10], supercapacitors [11] and batteries [12], but their applications in electrochemical sensing have been reported much less in the literature [13].

On the other hand, both experimental and theoretical studies have demonstrated that the structural defects (edges, vacancies, voids, boundaries, etc.) play a crucial role in determining the catalyst activity since the electrochemical reactions are most prior to occur at the defective sites [14-16]. In this regard, the defect-engineering to introduce more surface defects on graphene can be taken as an effective strategy to boost its catalytic activity. Several approaches were proposed to create the graphene defects such as nanostructures regulation [15], chemical modification [17], plasma etching [18] and dopants removal [19], and obtained defect-rich graphene materials achieved an considerable enhancement in catalytic performances. Therefore, considering the aforementioned advantages of heteroatom dopants and structural defects, it is anticipated that the methods to introduce both target dopants and 
appropriate defects will mostly explore the potentially high catalytic activity of the graphene. However, as far as we are aware no previous study related to this topic for electrochemical sensing has been reported.

Phosphorus (P) is one of the N-group elements and shows similar chemical properties to $\mathrm{N}$ due to their same number of valence electrons [20]. It has been reported that $\mathrm{P}$-doped graphene (PG) exhibits a better catalytic activity than $\mathrm{N}$-doped graphene due to the larger atomic radius and higher electron-donating ability of $\mathrm{P}$ dopants [20]. Although the PG is extensively investigated for energy-relevant electrochemical applications[21-23], its sensing performances are still rarely evaluated [24]. In addition, plasma-assisted technique is an efficient tool for surface modification of the materials due to the high activity of charged particles in plasma (positive/negative ions, electrons, free radicals and reactive species) [25]. Plasma treatment has been recently confirmed as an effective approach to etch the graphene surface and create high amounts of topological defects without worsening its intrinsic properties [18, 26, 27].

Herein, we developed a unique P-doped and plasma-etched graphene (abbreviated as PG-E) as an efficient metal-free catalyst for electrochemical sensing. P-doping of graphene was realized by the combined hydrothermal/annealing methods, and following plasma treatment was adopted to produce more structural defects on graphene, as illustrated in Scheme 1. The morphology and structure of as-prepared PG-E were comprehensively characterized. As a proof of concept, the PG-E was then applied for metal-free dopamine (DA) sensing. Owing to the synergic effect of 
introduced P-dopants and plasma-engineered defects, the PG-E modified electrode was found to show substantially improved activity for electrocatalytic oxidation of DA and good sensing performances for sensitive and selective detection of DA.

\section{Experimental}

\subsection{Synthesis of PG-E}

All the reagents were purchased from Lanzhou New Wanke, Ltd. and used as received without further purification. Graphene oxide (GO) was prepared from graphite powders (200 $\mu \mathrm{m}, 99.9 \%$ purity) using a modified Hummer's method [28]. PG was synthesized by a hydrothermal method followed by annealing treatment [24]. In brief, $50 \mathrm{~mL}$ of $\mathrm{GO}$ aqueous dispersion $\left(1 \mathrm{mg} \cdot \mathrm{mL}^{-1}\right)$ was first mixed with $20 \mu \mathrm{L}$ of phytic acid solution $(60 \mathrm{wt} \%)$ by ultrasonication for $10 \mathrm{~min}$. Afterwards, the homogeneous solution was transferred into a $100 \mathrm{~mL}$ of Teflon-lined autoclave and heated at $180{ }^{\circ} \mathrm{C}$. After reaction for $12 \mathrm{~h}$, the autoclave was cooled to room temperature and the resultant precipitates were collected and washed with ethanol and water several times, followed by drying in an oven at $60{ }^{\circ} \mathrm{C}$ for $12 \mathrm{~h}$. Finally, the obtained powders were annealed at $850{ }^{\circ} \mathrm{C}$ for $2 \mathrm{~h}$ under $\mathrm{N}_{2}$ atmosphere to prepare PG. For comparison, undoped reduced graphene oxide (RGO) was also synthesized without adding phytic acid during the hydrothermal process.

The plasma etching was carried out on a commercial AX-1000 plasma equipment (ADTEC Ltd.) with a radio-frequency (RF, $13.56 \mathrm{MHz})$ power generator. The as-synthesized samples were treated with Ar plasma for 40 min at a RF power level of $200 \mathrm{~W}$. The plasma-etched PG sample is labeled as PG-E. For comparison, 
reduced graphene oxide (RGO) etched by plasma was also synthesized and labeled as RGO-E.

\subsection{Characterizations and Electrochemical measurements}

Transmission electron microscopy (TEM), high-resolution transmission electron microscopy (HRTEM) and scanning transmission electron microscopy (STEM) were conducted on a JEM-2100 (JEOL Ltd.) microscope. X-ray diffraction (XRD) pattern was recorded on a 7000LX powder diffractometer (Shimadzu Ltd.). X-ray photoelectron spectroscopy (XPS) analysis was carried out on a PHI 5702 spectrometer (Perkin-Elmer Ltd.). Raman spectra were recorded using an Alpha 300R Raman spectroscope (WITEC Ltd.). Nitrogen adsorption/desorption isotherms were conducted on an ASAP 2020 instrument (Micromeritics Ltd.).

All the electrochemical measurements (electrochemical impedance spectroscopy (EIS), cyclic voltammetry (CV), differential pulse voltammetry (DPV) and amperometry) were conducted on a PGSTAT-302N electrochemical workstation (Metrohm Ltd.) using a conventional three-electrode system at room temperature. An $\mathrm{Ag} / \mathrm{AgCl}$ electrode and a $\mathrm{Pt}$ wire were used as reference and counter electrodes, respectively. A modified glassy carbon electrode (GCE, $3 \mathrm{~mm}$ in diameter) was used as a working electrode. To prepare the working electrode, each catalyst was firstly mixed with a dimetbylformamide (DMF) solution $\left(1 \mathrm{mg} \cdot \mathrm{mL}^{-1}\right)$ to form a homogeneous ink. The catalyst ink $(10 \mu \mathrm{L})$ was then drop-cast onto the surface of GCE and followed by drying at room temperature.

\section{Results and discussion}




\subsection{Characterizations of PG-E}

The morphologies and microstructures of as-prepared samples were characterized by TEM. It is shown in Fig. 1a that the RGO presents a transparent and wavy morphology, suggesting the well exfoliated or thin layered graphene nanosheets. This can also be reflected from the HRTEM image (Fig. S1a, Supplementary Information $\uparrow(\mathrm{SI} \uparrow)$ ), showing that the RGO nanosheets consist of 3-5 graphene layers. After P-doping, as shown in Fig. 1b, the PG exhibits more ripples and wrinkles than RGO, which is attributed to the distorted carbon structure of graphene induced by P-doping [29]. STEM images (Fig. 1c) clearly indicate the presence of C, O, and P elements in PG and P elements are homogeneously distributed throughout the PG, suggesting that the $\mathrm{P}$ atoms are successfully incorporated into the graphene frameworks. With further plasma treatment, the PG-E (Fig. 1d) displays a number of nanosized holes on the basin surface of graphene probably resulting from the plasma-etched structure defects, while such nanosized holes are almost absent on original PG under the same TEM magnification (Fig. S1b, SI $\uparrow$ ). In addition, the corresponding selected area electron diffraction (SAED) pattern (Fig. 1e) of PG-E reveals intense concentric diffraction rings while the SAED pattern of PG (Fig. S1c, SI†) displays obvious hexagonal spots, attesting that the PG-E has more structural defects [30]. The defective structure of PG-E can also be corroborated from the XRD (Fig. S2, SI†) and following Raman/XPS analysis. Moreover, the graphitic crystalline structures of PG-E (Fig. S1d, SI†) remain relatively unchanged as compared to RGO (Fig. S1a, SI†), indicating the plasma etching does not change the intrinsic graphene 
structures, consistent with the other reports $[18,26]$.

Raman spectroscopy was used to gain information about the graphene structural defects. It is seen in Fig. 2a that the Raman spectra of RGO, PG and PG-E display a G-band at $\sim 1590 \mathrm{~cm}^{-1}$ and a D-band at $\sim 1350 \mathrm{~cm}^{-1}$, corresponding to the graphitic carbons and structural defects, respectively[31, 32]. The $I_{\mathrm{D}} / I_{\mathrm{G}}$ intensity ratio can give a quantitative estimation of defect density in graphene, which increases in the order of RGO (0.98) < PG (1.09) < PG-E (1.38), suggesting that P-doping and plasma-etching can both lead to the increased defects in graphene. It is noteworthy that the ID/IG increment caused by P-doping is only about one third that by plasma-etching, suggesting that the plasma-etching is more powerful than P-doping to produce structural defects on graphene. This can be explained that the heteroatom doping is primarily realized through occupying vacant or edged sites by dopants[33], which can only cause the distortion in carbon lattice, whereas the plasma-etching is able to directly remove a small portion of $\mathrm{C}$ atoms (etching effect) resulting in a high level of defects as the form of etched nanoholes (Fig. 1d).

XPS analysis was performed to probe the chemical structures of the samples. It is shown in Fig. $2 b$ that the survey scans of PG and PG-E exhibit the peaks of C 1s, O 1s, and $\mathrm{P} 2 \mathrm{p} / \mathrm{P} 2 \mathrm{~s}$ (Fig. 2b), while the $\mathrm{P} 2 \mathrm{p} / \mathrm{P}$ 2s peaks are absent in RGO, suggesting once again the successful P-doping of graphene. Based on the XPS quantitative analysis (Table S1, SI $\dagger$ ), the P concentrations are 2.4 at.\% and 2.1 at.\% for PG and PG-E, respectively. The slightly decreased $\mathrm{P}$ concentration in PG-E is due possibly to the somewhat loss of $\mathrm{P}$ dopants during the plasma-etching process. Fig. 2c shows the 
high-resolution P 2p spectra of PG and PG-E. The P 2p peak can be deconvoluted into two peaks at binding energies of $131.3 \mathrm{eV}$ and $133.6 \mathrm{eV}$, which are assigned to $\mathrm{P}-\mathrm{C}$ and $\mathrm{P}-\mathrm{O}$ bonds [22], respectively. The relative percentages of $\mathrm{P}-\mathrm{C}$ and $\mathrm{P}-\mathrm{O}$ in $\mathrm{PG}-\mathrm{E}$ change little compared to those in PG (Table S1, SI†), indicating the plasma-etching has little effect on the P bonding configurations. Besides, the deconvoluted $\mathrm{C}$ 1s spectra (Fig. S3 \& Table S1, SI†) show the increased $\mathrm{sp}^{3} / \mathrm{sp}^{2}$ ratio (0.386) in PG-E with respect to PG (0.281), further confirming that more defective sites are yielded in PG-E [18]. Moreover, the $\mathrm{N}_{2}$ adsorption-desorption measurements (Fig. 2d) show the comparable BET surface areas of PG $\left(288 \mathrm{~m}^{2} \cdot \mathrm{g}^{-1}\right)$ and PG-E $\left(296 \mathrm{~m}^{2} \cdot \mathrm{g}^{-1}\right)$. The pore size distribution curves (Fig. 2d, inset) reveal a slight increase in mesopores populations in PG-E as compared to PG, presumably arising from the formation of plasma-etched nanoholes, as observed in Fig. 1d. A certain amount of mesopores are beneficial for the eletrocatalytic activity as they can sever as the channels for the favorable diffusion of reactants and electrolytes [34].

\subsection{Evaluation of PG-E for DA sensing}

Dopamine, an important neurotransmitter, plays a crucial role in functioning the mammalian neurophysiological systems [35-37]. The abnormality of the DA levels can lead to a series of illnesses and symptoms, such as schizophrenia, Parkinson's disease, HIV infection, depression, drug addiction, etc[38]. Thus, the development of efficient electrochemical sensors for highly sensitive and selective detection of DA is of great importance in neurophysiological clinical diagnosis. In the construction of DA electrochemical sensors, the electrocatalytic oxidation of DA (EOD) is a critical 
reaction in determining the DA sensing performances, and it is a key to develop a highly active catalyst to promote the EOD activity and improve the DA sensitivity [38, 39]. Electrochemical characterizations were then carried out to evaluate the EOD activity of the electrodes modified with different catalysts to understand the combined effect of P-dopants and plasma-engineered defects.

Fig. 3a shows the CVs of bare GCE, RGO/GCE, PG/GCE and PG-E/GCE electrodes toward EOD in PBS (0.1 M, pH 7.0) containing 0.5 mM DA. As seen, bare GCE exhibits a negligible EOD activity as evidenced by the weak peak current $\left(I_{\mathrm{p}}\right)$ and broad peak-to-peak separation $(\Delta E)$, whereas the RGO/GCE shows a slightly improved EOD activity due to the strong $\pi-\pi$ interactions between the phenyl moiety of DA molecule and $\pi$-conjugation of graphene[40, 41]. In addition, the PG/GCE presents a noticeably higher $I_{\mathrm{p}}$ and narrower $\Delta E$ compared to RGO/GCE, suggesting the much improved EOD activity as a result of P-doping, correlating well with the previous reports associated with the $\mathrm{N}$-doping[42] and S-doping[43] for improving the EOD activity of graphene. Notably, after plasma treatment, the PG-E/GCE exhibits the best EOD activity with the highest $I_{\mathrm{p}}$ and narrowest $\Delta E$. This clearly reveals that introduction of P-dopants, coupled with plasma-induced defects, can significantly improve the EOD activity of graphene. It is worth noting that the electrodes modified with RGO-E (etched RGO but without $\mathrm{P}$ doping) exhibits an inferior catalytic activity to PG-E/GCE (Fig. S4, SI†), signifying that the synergistic effect of P dopants and topological defects leads to the considerably enhanced EOD activity, consistent well with the previous results obtained in other electrochemical 
reactions for applications in water splitting [44] and supercapacitors [26].

The amount of active sites is highly associated with the catalytic activity[8]. Given that only accessible sites are active for the electrocatalysis reactions[6], the electrochemically surface area (ECSA) capable of reflecting the actual amount of accessible active sites can be determined from the electrochemical behavior of $\left[\mathrm{Fe}(\mathrm{CN})_{6}\right]^{3-} /^{4-}$ at the modified electrode according to the Randles-Sevcik equation[45]. Fig. S5 (SI $\dagger$ ) shows the representative plot for the determination of ECSA. Based on the slopes of the linear fitted plots (Fig. 3b), the calculated ECSA values increase in the order of RGO/GCE $\left(0.11 \mathrm{~cm}^{2}\right)<\operatorname{PG} / \mathrm{GCE}\left(0.27 \mathrm{~cm}^{2}\right)<$ PG-E/GCE $\left(0.48 \mathrm{~cm}^{2}\right)$, indicating that the combination of $\mathrm{P}$ dopants and plasma-etched defects offers the large amount of active centers for DA adsorption and electrocatalytic oxidation, which dramatically boost the EOD activity.

Aside from the increased accessible active sites, the superior EOD activity of PG-E is believed to originate from the improved electron transfer capability caused by the combined effect of P-doping with enriched defects. EIS measurement was conducted to examine the electron-transfer properties. Fig. 3(c) shows the EIS spectra of RGO/GCE, PG/GCE and PG-E/GCE electrodes. The impedance data can be fitted well by an equivalent circuit model (Fig. 3(c), inset), and the charge transfer resistance $\left(R_{\mathrm{ct}}\right)$ can be estimated from the semicircle diameter in the high-frequency region. Noticeably, the PG-E/GCE presents the much lower $R_{\text {ct }}(58.6 \Omega)$ than

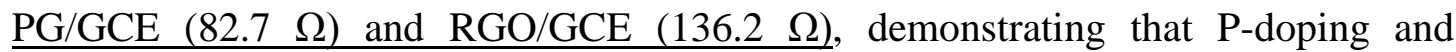
structural defects can both effectively lower the charge transfer resistance of the 
electrochemical reactions. This can be explained that $\mathrm{P}$ dopants can increase the charge mobility of carbon because of its electron-donor properties[46], while the defects are able to enhance the density of state (DOS) resulting in a high carrier concentration[47]. Both factors facilitate the fast electron transport across the catalyst-GCE interface for accelerating the electrochemical reaction kinetics. Therefore, it can be concluded that introducing both topological defects and P-dopants in graphene can synergistically lead to the significantly elevated EDO activity of graphene by increasing the accessible active sites and promoting the electron transport capability. Moreover, the CVs of PG-E/GCE with the presence of DA at different scan rates reveal that the peak current is linearly proportional to the scan rate (Fig. 3d), indicating that the EDO on the PG-E/GCE electrode is an adsorption-controlled electrochemical process. The perfect linear response to scan rate is another indication of fast electron transfer efficiency for the EDO reaction process[38, 48].

The outstanding EGO activity makes the PG-E modified electrode a promising sensor for DA detection. DPV technique was employed to quantitatively assess the DA sensing performances of PG-E/GCE. Fig. 4a shows the DPV curves of PG-E/GCE in PBS (0.1 M, pH 7.0) containing different concentrations of DA (0.1-160 $\mu \mathrm{M})$. A group of well-defined oxidation peaks of DA are observed at $0.18 \mathrm{~V}$. The calibration plot (Fig. 4b) shows that the DPV peak current is linear with the DA concentration with a wide linear range of $0.1-120 \mu \mathrm{M}$. From the slope of the calibration plot, the limit of detection (LOD) is determined to be $0.006 \mu \mathrm{M}$ at a signal-to-noise ratio of 3 . Such sensing performances are better than those of most 
carbon-based metal-free catalysts and even superior or comparable to those of most recently reported metal-based catalysts (Table $\mathrm{S} 2, \mathrm{SI} \uparrow$ ), suggesting that the present PG-E is a promising metal-free electrocatalyst for DA sensing. It is demonstrated that the significantly enhanced EGO activity of PG-E resulting from the synergetic effect of P-dopants and plasma-engineered defects contributes to the excellent DA sensing performances of PG-E/GCE.

The foreign species of uric acid (UA) and ascorbic acid (AA) are normally co-existed with DA in the human serum, and it is difficult to identify DA in the mixture because their oxidation potentials greatly overlap[40]. The interference study was then conducted by DPV testing in PBS (0.1 M, pH 7.0) containing a ternary mixture of $0.2 \mathrm{mM} \mathrm{AA}, 0.04 \mathrm{mM}$ DA and $0.05 \mathrm{mM}$ UA. As shown in Fig. 4c, one can observe three well-resolved peaks with marked peak separations of $185 \mathrm{mV}$ (AA-DA) and $128 \mathrm{mV}$ (DA-UA), indicating the PG-E/GCE is able to simultaneously distinguish DA, UA and AA. In addition, as seen in Fig. 4d, the amperometric response of PG-E/GCE to DA remains almost unchanged after the sequential addition of glucose (Glu), UA, AA, lactose (Lac) and fructose (Fru). The long-term stability of PG-E/GCE was investigated by measuring the CV of DA after 10 days storage. It is seen in Fig. 4e that the CV curve shows a negligible difference compared with the initial one. Furthermore, as shown in Fig. 4f, the PG-E/GCE retains $95.6 \%$ its initial amperometric current after running time of 3000s. These results prove that the PG-E/GCE possesses the extraordinary selectivity and stability for DA detection.

To verify the applicability of the present sensor, the PG-E/GCE was then 
employed to detect the concentrations of DA in human serum samples using a standard adding method[39]. All the samples were diluted with PBS (0.1 M, pH 7.0) and each determination is the average of three measurements. The detecting results are listed in Table S3. It is found that the recoveries for the determination of DA are within the acceptable range of $98.5-103.2 \%$, demonstrating that the present PG-E/GCE sensor can be practically utilized for the accurate detection of DA in the real samples.

\section{Conclusions}

In summary, the PG-E with both P-dopants and plasma-engineered defects was successfully synthesized by the combined hydrothermal/annealing and followed plasma treatment, as confirmed by the microscopic and spectroscopic techniques. Electrochemical measurements indicated that the PG-E modified electrode exhibited a dramatically improved EOD activity, which could be ascribed to the synergistic effect of P-dopants and plasma-etched defects that led to the increased active sites and enhanced electron transport. The PG-E based DA sensor showed excellent sensing performances with a low detection limit of $0.006 \mu \mathrm{M}$ and a wide linear range of $0.1-120 \mu \mathrm{M}$, which are superior or comparable to those of most recently reported metal-free and metal-based catalysts. The PG-E sensor also displayed high selectivity, good stability, and accurate analysis in real samples, making PG-E a promising metal-free electrocatalyst for DA sensing. We believe that that there is still a room to further enhance the EOD activity and sensing performances of PG-E by optimizing the processing of P-doping and plasma treatment, which are currently being 
investigated. The present work may provide a new strategy to design and prepare highly active graphene-based metal-free for electrochemical sensing applications.

\section{Acknowledgement}

This work is supported by National Science Foundation of China (51501083), Gansu Provincial Science and Technology Department Fund (1606RJZA097), Gansu Provincial Education Department Fund (2015A-061), Lanzhou Jiaotong University Youth Fund (2014015), Science and Technology Department of Hebei Province Projects (152111230), and Health Department of Hebei Province Project (20160030).

\section{References}

[1] J.N. Tiwari, V. Vij, K.C. Kemp, K.S. Kim, Engineered carbon-nanomaterial based electrochemical sensors for biomolecules, ACS Nano, 10 (2016) 46-80.

[2] H. Beitollahi, H. Salimi, A triple electrochemical platform for simultaneous eetermination of isoproterenol, acetaminophen and tyrosine based on a glassy carbon electrode modified with hematoxylin and graphene, J. Electrochem. Soc., 163 (2016) 1157-1164.

[3] Y. Shao, J. Wang, H. Wu, J. Liu, I.A. Aksay, Y. Lin, Graphene based electrochemical sensors and biosensors: a review, Electroanalysis, 22 (2010) 1027-1036.

[4] J. Wang, Electrochemical biosensing based on noble metal nanoparticles, Microchim. Acta, 177 (2012) 245-270.

[5] X. Luo, A. Morrin, A.J. Killard, M.R. Smyth, Application of nanoparticles in electrochemical sensors and biosensors, Electroanalysis, 18 (2006) 319-326.

[6] X.W. Wang, G.Z. Sun, P. Routh, D.H. Kim, W. Huang, P. Chen, Heteroatom-doped graphene materials: syntheses, properties and applications, Chem. Soc. Rev., 43 (2014) 7067-7098.

[7] J.J. Duan, S. Chen, M. Jaroniec, S.Z. Qiao, Heteroatom-doped graphene-based materials for energy-relevant electrocatalytic processes, ACS Catal., 5 (2015) 5207-5234.

[8] A. Ambrosi, C.K. Chua, N.M. Latiff, A.H. Loo, C.H. Wong, A.Y. Eng, A. Bonanni, M. Pumera, Graphene and its electrochemistry-an update, Chem. Soc. Rev., 45 (2016) 2458-2493.

[9] S.E. Baghbamidi, H. Beitollahi, S. Tajik, Synthesis of graphene oxide nanosheets and its application

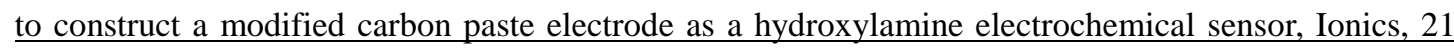
(2015) 1-8.

[10] X. Zhou, J. Qiao, L. Yang, J. Zhang, A review of graphene-based nanostructural materials for both catalyst supports and metal-free catalysts in PEM fuel cell oxygen reduction reactions, Adv. Energy Mater., 4 (2014) 1289-1295.

[11] Y. Deng, Y. Xie, K. Zou, X. Ji, Review on recent advances in nitrogen-doped carbons: preparations and applications in supercapacitors, J. Mater. Chem. A, 4 (2015) 1144-1173.

[12] R. Raccichini, A. Varzi, S. Passerini, B. Scrosati, The role of graphene for electrochemical energy 
storage, Nature Mater., 14 (2015) 271-279.

[13] J. Liu, Z. Liu, C.J. Barrow, W. Yang, Molecularly engineered graphene surfaces for sensing applications: A review, Anal. Chim. Acta, 859 (2015) 1-19.

[14] A.L. Shen, Y.Q. Zou, Q. Wang, R.A. Dryfe, X.B. Huang, S. Dou, L.M. Dai, S.Y. Wang, Oxygen reduction reaction in a droplet on graphite: Direct evidence that the edge is more active than the basal plane, Angew. Chem. Int. Edit., 53 (2014) 10804-10808.

[15] Y.F. Jiang, L.J. Yang, T. Sun, J. Zhao, Z.Y. Lyu, O. Zhuo, X.Z. Wang, Q. Wu, J. Ma, Z. Hu, Significant contribution of intrinsic carbon defects to oxygen reduction activity, ACS Catal., 5 (2015) 6707-6712.

[16] C. Tang, H.F. Wang, X. Chen, B.Q. Li, T.Z. Hou, B.S. Zhang, Q. Zhang, M.M. Titirici, F. Wei, Topological defects in metal-free nanocarbon for oxygen electrocatalysis, Adv. Mater., 28 (2016) 6845-6851.

[17] Y. Hu, D. He, Y. Wang, M. Fu, X. An, X. Zhao, Defect-introduced graphene sheets with hole structure as lithium-ion battery anode, Mater. Lett., 164 (2016) 278-281.

[18] L. Tao, Q. Wang, S. Dou, Z.L. Ma, J. Huo, S.Y. Wang, L.M. Dai, Edge-rich and dopant-free graphene as a highly efficient metal-free electrocatalyst for the oxygen reduction reaction, Chem. Commun., 52 (2016) 2764-2767.

[19] Y. Jia, L. Zhang, A. Du, G. Gao, J. Chen, X. Yan, C.L. Brown, X. Yao, Defect graphene as a trifunctional catalyst for electrochemical reactions, Adv. Mater., 28 (2016) 9532-9538.

[20] P. Karthika, N. Rajalakshmi, K. Dhathathreyan, Phosphorus-doped exfoliated graphene for supercapacitor electrodes, J. Nanosci. Nanotechno., 13 (2013) 1746-1751.

[21] R. Li, Z. Wei, X. Gou, W. Xu, Phosphorus-doped graphene nanosheets as efficient metal-free oxygen reduction electrocatalysts, RSC Adv., 3 (2013) 9978-9984.

[22] C. Zhang, N. Mahmood, H. Yin, F. Liu, Y. Hou, Synthesis of phosphorus-doped graphene and its multifunctional applications for oxygen reduction reaction and lithium ion batteries, Adv. Mater., 25 (2013) 4932-4937.

[23] Y. Wen, B. Wang, C. Huang, L. Wang, D. Hulicova-Jurcakova, Synthesis of phosphorus-doped graphene and its wide potential window in aqueous supercapacitors, Chem. Eur. J., 21 (2015) 80-85.

[24] Y. Tian, Z. Wei, K. Zhang, S. Peng, X. Zhang, W. Liu, K. Chu, Three-dimensional phosphorus-doped graphene as an efficient metal-free electrocatalyst for electrochemical sensing, Sensor. Actuat. B Chem., 241 (2017) 584-591.

[25] W. Qi, X.K. Wang, Z.F. Chai, W.P. Hu, Low-temperature plasma synthesis of carbon nanotubes and graphene based materials and their fuel cell applications, Chem. Soc. Rev., 42 (2014) 8821-8834.

[26] J. Zhu, A.S. Childress, M. Karakaya, S. Dandeliya, A. Srivastava, Y. Lin, A.M. Rao, R. Podila, Defect-engineered graphene for high-energy- and high-power-density supercapacitor devices, Adv. Mater., 28 (2016) 7185-7192.

[27] Y. Tian, R. Mei, D.Z. Xue, X. Zhang, W. Peng, Enhanced electrocatalytic hydrogen evolution in graphene via defect engineering and heteroatoms co-doping, Electrochim. Acta, 219 (2016) 781-789.

[28] D. Li, M.B. Müller, S. Gilje, R.B. Kaner, G.G. Wallace, Processable aqueous dispersions of graphene nanosheets, Nature Nanotechnol., 3 (2008) 101-105.

[29] A.G. Kannan, J.X. Zhao, S.G. Jo, Y.S. Kang, D.W. Kim, Nitrogen and sulfur co-doped graphene counter electrodes with synergistically enhanced performance for dye-sensitized solar cells, J. Mater. Chem. A, 2 (2014) 12232-12239.

[30] H.B. Wang, T. Maiyalagan, X. Wang, Review on recent progress in nitrogen-doped graphene: 
synthesis, characterization, and its potential applications, ACS Catal., 2 (2012) 781-794.

[31] A.C. Ferrari, J.C. Meyer, V. Scardaci, C. Casiraghi, M. Lazzeri, F. Mauri, S. Piscanec, D. Jiang, K.S. Novoselov, S. Roth, Raman spectrum of graphene and graphene layers, Phys. Rev. Lett., 97 (2006) 187401.

[32] K. Chu, C. Jia, Enhanced strength in bulk graphene-copper composites, Phys. Status Solidi (A), 211 (2014) 184-190.

[33] Y. Liu, Y. Shen, L. Sun, J. Li, C. Liu, W. Ren, F. Li, L. Gao, J. Chen, F. Liu, Y. Sun, N. Tang, H.-M. Cheng, Y. Du, Elemental superdoping of graphene and carbon nanotubes, Nat. Commun., 7 (2016) 10921.

[34] Y.P. Zhu, Y. Liu, Y.P. Liu, T.Z. Ren, T. Chen, Z.Y. Yuan, Direct synthesis of phosphorus-doped mesoporous carbon materials for efficient electrocatalytic oxygen reduction, ChemCatChem, 7 (2015) 2903-2909.

[35] S. Jahani, H. Beitollahi, Selective detection of dopamine in the presence of uric acid using NiO nanoparticles decorated on graphene nanosheets modified screen-printed electrodes, Electroanalysis, 28 (2016) 2022-2028.

[36] T. Łuczak, Preparation and characterization of the dopamine film electrochemically deposited on a gold template and its applications for dopamine sensing in aqueous solution, Electrochim. Acta, 53 (2008) 5725-5731.

[37] E. Molaakbari, A. Mostafavi, H. Beitollahi, Simultaneous electrochemical determination of dopamine, melatonin, methionine and caffeine, Sensor. Actuat. B Chem., 208 (2015) 195-203.

[38] A. Pandikumar, T.P. See, F.S. Omar, S. Jayabal, K.Z. Kamali, N. Yusoff, A. Jamil, R. Ramaraj, S.A. John, N.L. Hong, Graphene and its nanocomposite material based electrochemical sensor platform for dopamine, RSC Adv., 4 (2014) 63296-63323.

[39] H. Beitollahi, I. Sheikhshoaie, Novel nanostructure-based electrochemical sensor for simultaneous determination of dopamine and acetaminophen, Mater. Sci. Eng C, 32 (2012) 375-380.

[40] Z.H. Sheng, X.Q. Zheng, J.Y. Xu, W.J. Bao, F.B. Wang, X.H. Xia, Electrochemical sensor based on nitrogen doped graphene: simultaneous determination of ascorbic acid, dopamine and uric acid, Biosens. Bioelectron., 34 (2012) 125-131.

[41] H. Beitollahi, F. Garkani Nejad, Graphene oxide/ZnO nano composite for sensitive and selective electrochemical sensing of levodopa and tyrosine using modified graphite screen printed electrode, Electroanalysis, 28 (2016) 2237-2244.

[42] X. Feng, Y. Zhang, J. Zhou, Y. Li, S. Chen, L. Zhang, Y. Ma, L. Wang, X. Yan, Three-dimensional nitrogen-doped graphene as an ultrasensitive electrochemical sensor for the detection of dopamine, Nanoscale, 7 (2015) 2427-2432.

[43] M. Li, C. Liu, H. Zhao, H. An, H. Cao, Y. Zhang, Z. Fan, Tuning sulfur doping in graphene for highly sensitive dopamine biosensors, Carbon, 86 (2015) 197-206.

[44] Y. Ito, W.T. Cong, T. Fujita, Z. Tang, M.W. Chen, High catalytic activity of nitrogen and sulfur co-doped nanoporous graphene in the hydrogen evolution reaction, Angew. Chem. Int. Edit., 54 (2015) 2131-2136.

[45] K.B. Oldham, Analytical expressions for the reversible Randles-Sevcik function, J. Electroanal. Chem. Inter. Electrochem., 105 (1979) 373-375.

[46] X. Ma, G. Ning, C. Qi, C. Xu, J. Gao, Phosphorus and nitrogen dual-doped few-layered porous graphene: a high-performance anode material for lithium-ion batteries, ACS Appl. Mater. Inter., 6 (2014) 14415-14422. 
[47] J. Chen, Y. Han, X. Kong, X. Deng, H.J. Park, Y. Guo, S. Jin, Z. Qi, Z. Lee, Z. Qiao, The origin of improved electrical double-layer capacitance by inclusion of topological defects and dopants in graphene for supercapacitors, Angew. Chem. Int. Edit., 55 (2016) 13822-13827.

[48] H. Beitollahi, A. Mohadesi, S. Mohammadi, A. Pahlavan, H. Karimi-Maleh, A. Akbari, New voltammetric strategy for determination of dopamine in the presence of high concentrations of acetaminophen, folic acid and N-acetylcysteine, J. Mol. Liq., 169 (2012) 130-135. 


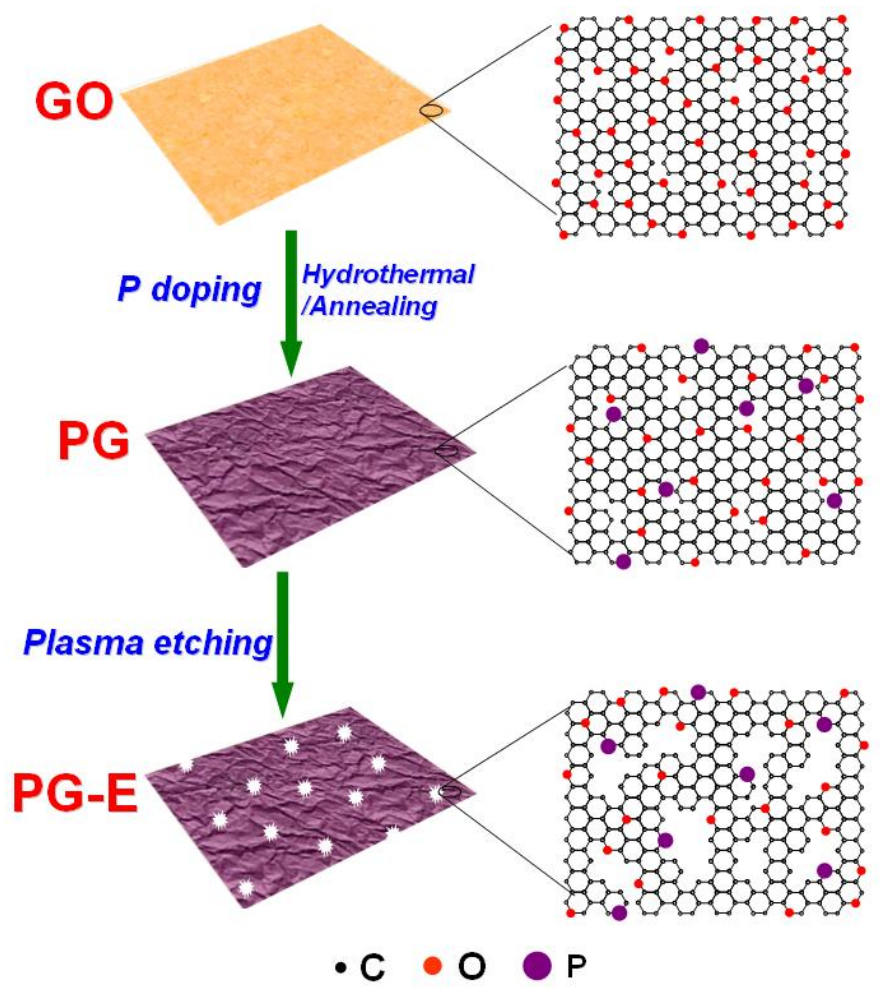

Scheme 1. Illustration of the synthesis of PG-E. 

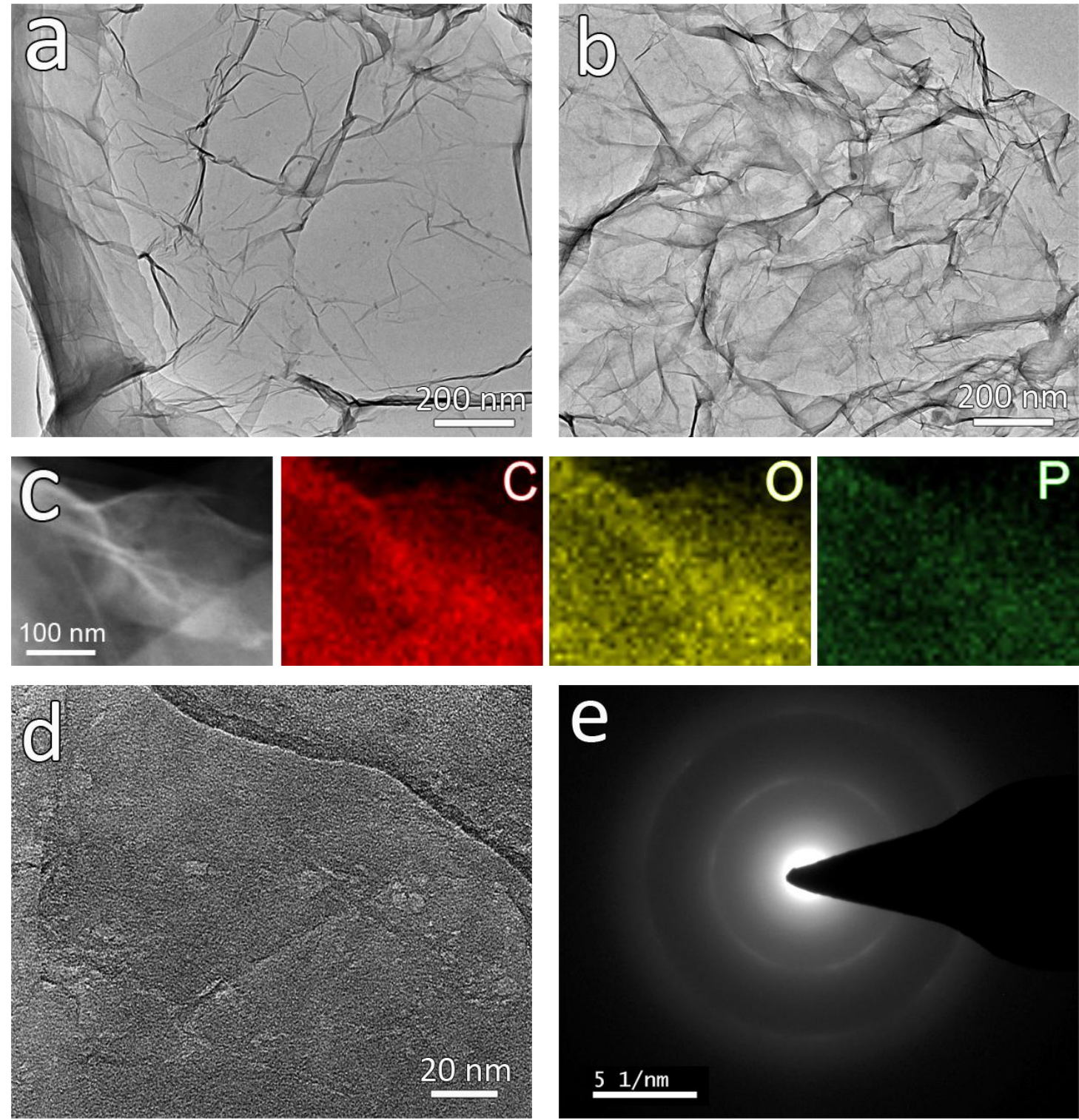

Fig. 1. TEM images of (a) RGO and (b) PG. (c) STEM image of PG and corresponding elemental mapping images of $\mathrm{C}$, $\mathrm{O}$, and P. (d) Magnified TEM image of PG-E and corresponding SAED pattern (e). 

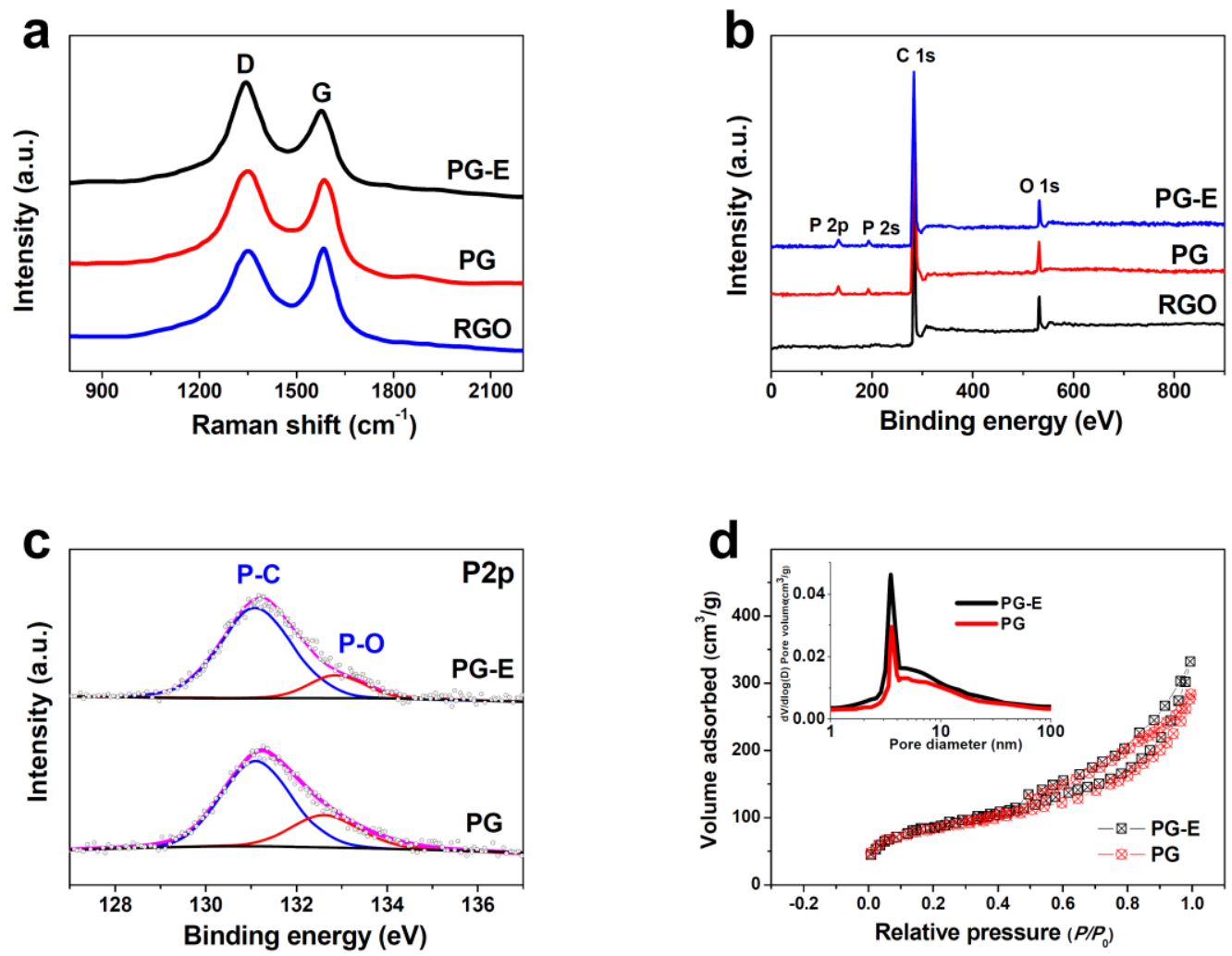

Fig. 2. (a) Raman spectra of RGO, PG and PG-E. (b) XPS survey scans of RGO, PG and PG-E. (c) High-resolution P 2p spectra of PG and PG-E. (D) Nitrogen adsorption/desorption isotherm curves of PG and PG-E and their corresponding pore size distributions (inset). 

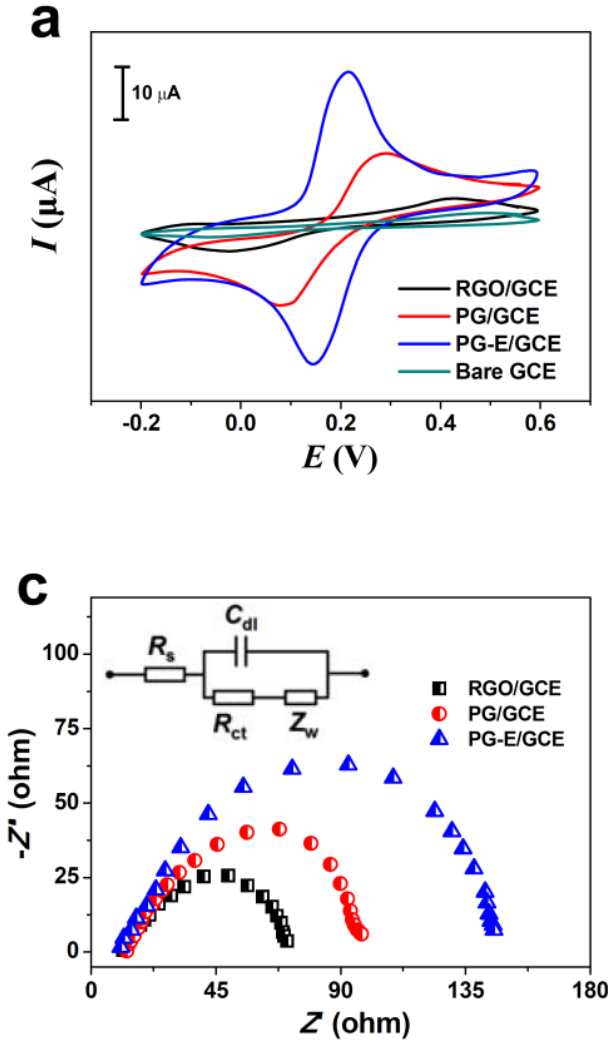
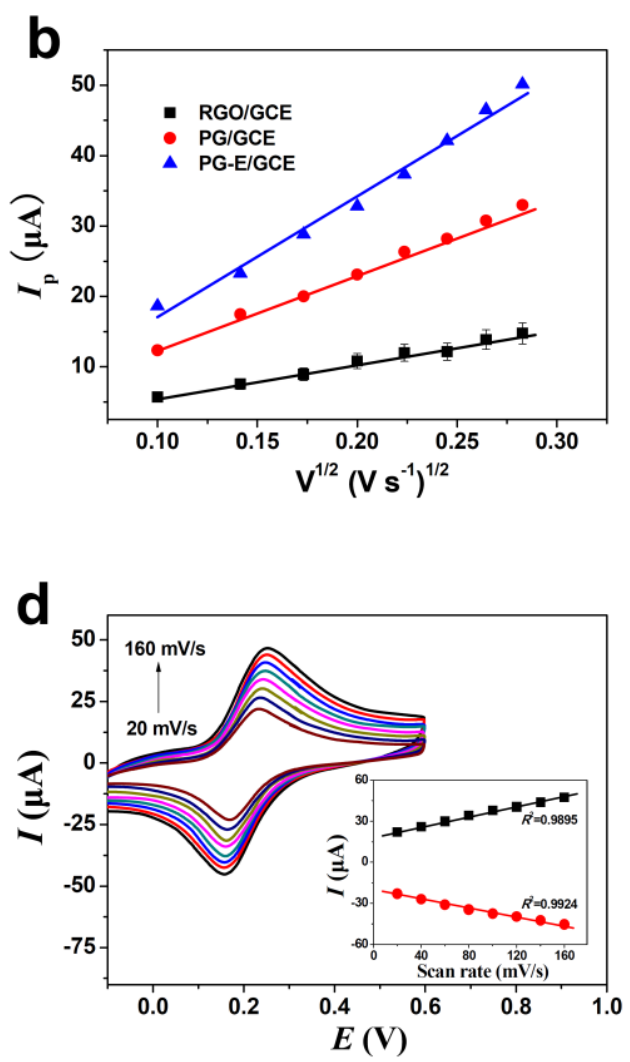

Fig. 3. (a) CVs of bare GCE, RGO/GCE, PG/GCE and PG-E/GCE in PBS (0.1 M, pH 7.0) containing $0.5 \mathrm{mM} \mathrm{DA}$ at the scan rate of $50 \mathrm{mV} / \mathrm{s}$. (b) Plots of peak potentials $\left(I_{\mathrm{p}}\right)$ vs. square root of the scan rate $\left(v^{1 / 2}\right)$ (Fig. S5, SI $\dagger$ ) to extract the ECSA values of RGO/GCE, PG/GCE and PG-E/GCE. (c) EIS spectra of RGO/GCE, PG/GCE and PG-E/GCE in $0.1 \mathrm{M} \mathrm{KCl}$ solution containing $5.0 \mathrm{mM}\left[\mathrm{Fe}(\mathrm{CN})_{6}\right]^{3-/ 4-}$. The inset is the equivalent circuit where $C_{\mathrm{dl}}$ is double layer capacitances, $R_{\mathrm{S}}$ is the solution resistance, $R_{\mathrm{ct}}$ is the charge transfer resistance and $Z_{\mathrm{w}}$ is the Warburg impedance. (d) CVs of PG-E/GCE in PBS (0.1 M, pH 7.0) containing $0.5 \mathrm{mM}$ at different scan rates of 20-160 mV/s (inset: plot of peak current $\left(I_{\mathrm{p}}\right)$ versus scan rate). 

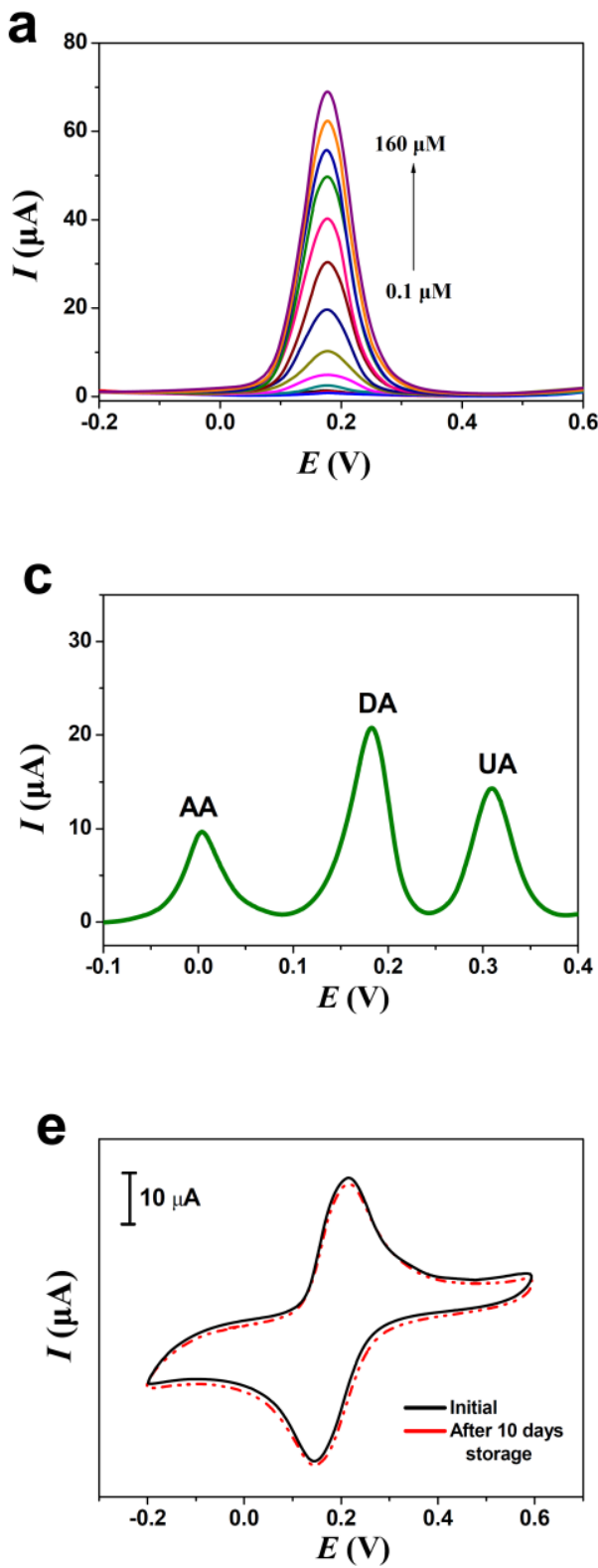
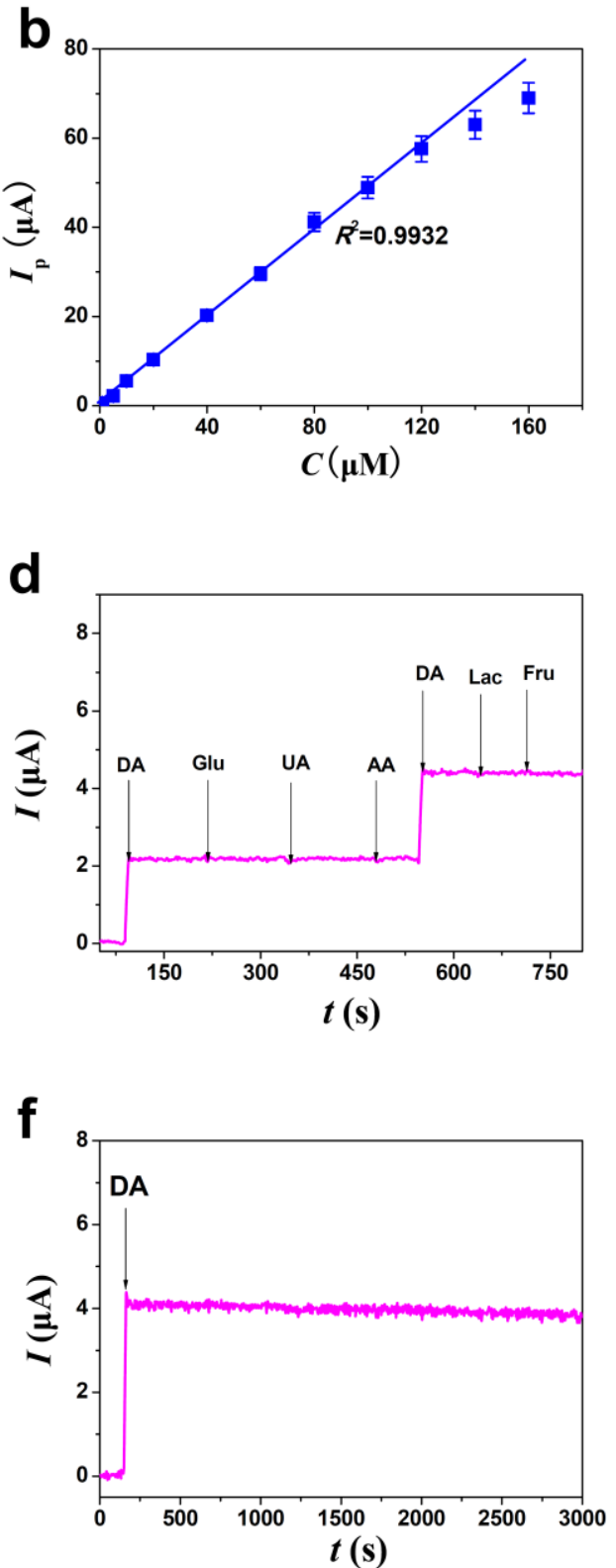

Fig. 4. (a) DPV responses of PG-E/GCE in PBS (0.1 M, pH 7.0) containing different concentrations of DA $(0.1-160 \mu \mathrm{M})$. (b) Calibration curve of DPV peak current $\left(I_{\mathrm{p}}\right)$ versus DA concentration $(C)$. (c) DPV response of PG-E/GCE in PBS (0.1 M, pH 7.0) containing $0.2 \mathrm{mM}$ AA, $0.04 \mathrm{mM}$ DA and $0.05 \mathrm{mM}$ UA. (d) Amperometric responses of PG-E/GCE with successive addition of $0.01 \mathrm{mM}$ DA and each $0.1 \mathrm{mM}$ of glucose (Glu), UA, AA, lactose (Lac) and fructose (Fru) in PBS (0.1 M, pH 7.0) at the applied potential of $0.18 \mathrm{~V}$. (e) $\mathrm{CV}$ responses of PG-E/GCE initially and after 10 days storage. (f) Amperometric response of PG-E/GCE with addition of $0.02 \mathrm{mM}$ DA after 3000s running time. 